\title{
COX-2/NEGF-Dependent Facilitation of Tumor-Associated Angiogenesis and Tumor Growth In Vivo
}

\author{
Satoko Yoshida, Hideki Amano, Izumi Hayashi, Hidero Kitasato, Mariko Kamata, \\ Madoka Inukai, Hirokuni Yoshimura, and Masataka Majima
}

Departments of Pharmacology (SY, HA, IH, MK, MI, MM), Thoracic Surgery (SY, HA, HY), and Microbiology (HK), Kitasato University School of Medicine, and Department of Molecular Pharmacology (SY, HA, IH, MM), Kitasato University Graduate School of Medical Sciences, Sagamihara, Japan

SUMMARY: Nonsteroidal anti-inflammatory drugs are known to suppress the occurrence and progression of malignancies such as colorectal cancers. However, the precise mechanism of these actions remains unknown. We have evaluated the role of an inducible cyclo-oxygenase (COX-2) in tumor-associated angiogenesis and tumor growth, and identified the downstream molecules involved using a ddy mouse model of sponge angiogenesis, which mimics tumor angiogenesis and is COX-2 and vascular endothelial growth factor (VEGF) dependent. In this model, VEGF expression was down-regulated by selective COX-2 inhibition with NS-398. To find out the involvement of COX-2/NEGF pathway in tumor-associated angiogenesis, we estimated angiogenesis occurring around implanted Millipore chambers containing sarcoma-180 (S-180) cells or Lewis lung carcinoma cells. Daily oral administration of NS-398 or of aspirin, a nonselective COX inhibitor, suppressed angiogenesis seen around the Millipore chambers. S-180 cells implanted in ddy mice formed substantial tumors with extensive angiogenesis markedly suppressed by aspirin and COX-2 inhibitors NS-398 and JTE522, but not by mofezolac, an inhibitor of constitutive COX-1. Tumor-associated angiogenesis was also significantly suppressed by a neutralizing antibody against VEGF. S-180 tumor growth in the subcutaneous tissues was also suppressed by aspirin, COX-2 selective inhibitors, and the VEGF antibody, but not by the COX-1 inhibitor. These results demonstrate that the inhibition of the COX-2/NEGF-dependent pathway was effective in tumor-associated angiogenesis, tumor growth, and tumor metastasis. (Lab Invest 2003, 83:1385-1394).

$N$ onsteroidal anti-inflammatory drugs (NSAIDs), which block the enzymatic activity of cyclooxygenase (COX), have been widely used for antiinflammatory and analgesic purposes. Early in the 1990s, it was reported that a significant reduction in mortality from colorectal cancer occurred depending on the cumulative doses of an NSAID, and further evidence suggested that NSAIDs also affect the incidence and progression of other types of cancer, pointing to a possible role of COX in other tumor formations (Smally and Dubois, 1997). Prostaglandins (PGs) belong to a group of oxygenated metabolites of arachidonic acid and are produced by the sequential

\section{DOI: 10.1097/01.LAB.0000090159.53224.B9}

Received April 7, 2003.

This work was supported by grants from an Integrative Research Program of the Graduate School of Medical Science, Kitasato University, and from Parents' Association Grant of Kitasato University School of Medicine. This work was also supported by a research grant (\#15390084), by a "High-tech Research Center" grant, and by a grant from The 21st Century COE Program, of Ministry of Education, Culture, Sports, Science and Technology (MEXT).

Address reprint requests to: Dr. Masataka Majima, Department of Pharmacology, Kitasato University School of Medicine, Kitasato 1-15-1, Sagamihara, Kanagawa 228-8555, Japan. E-mail: en3m-mjm@ asahi-net.or.jp actions of COX and specific PG synthases. Two COX isoforms have been identified: COX-1 is constitutively expressed in various tissues, whereas COX-2 is induced by mitogens, growth factors, cytokines, and tumor promoters. Using COX-2 knockout mice, it was revealed that a COX-2-dependent pathway increased the number and size of intestinal polyps generated by a mutation in the adenomatous polyposis coli $(A P C)$ gene. That report emphasized strongly the importance of COX-2 in colon tumor development (Ohshima et al, 1996). In spite of the efficacy of NSAIDs as anticancer agents, the precise mechanism or mechanisms of their antitumor action are not fully clarified and have given rise to wide-ranging discussion, in which some opinions are surprisingly unrelated to COX inhibition (Marx, 2001; Prescott et al, 1996; Shiff and Rigas, 1997).

Angiogenesis is an important factor in tumor development, and tumor-associated angiogenesis is mediated by the migration and proliferation of host endothelial cells. With the use of an in vitro coculture system comprising endothelial cells and colon carcinoma cells that overexpress COX-2, it was shown that the increased production of PGs by the carcinoma cells was associated with endothelial cell migration and tube formation (Tsujii et al, 1998). This suggested a role of COX-2 in tumor-induced angiogenesis. How- 
ever, the relevance of these in vitro observations to tumorigenesis in vivo remains to be established. For a mechanistic analysis of angiogenesis in vivo, we have developed a sponge implant model, in which a polyurethane sponge disk implanted subcutaneously in rats induces extensive angiogenesis in surrounding proliferative granuloma tissues (Hayashi et al, 2002; Majima et al, 1997, 2000; Muramatsu et al, 2000). With the use of this model, we have previously shown that angiogenesis occurs both concomitantly with the induction of COX-2 mRNA and is inhibited by administration either of a nonselective NSAID (indomethacin) or of selective COX-2 inhibitors (Majima et al, 2000). We further demonstrated that angiogenesis induced by either endogenous COX-2 or exogenous PGs was accompanied by increased expression of vascular endothelial growth factor (VEGF) and that angiogenesis was abolished by administration of an antisense oligonucleotide specific for VEGF mRNA in that model (Majima et al, 2000). These results suggest that COX2-catalyzed PG formation may mediate the tumorassociated angiogenesis and tumor growth in vivo in relation to VEGF expression.

In the present study, with the use of the compounds selective of COX isoforms and growth factor neutralizing antibodies, we have now identified the COX isoforms responsible for tumor-associated angiogenesis and tumor growth. Furthermore, we have characterized the downstream molecules responsible for COX-2-dependent PG formation. The present results will address the effectiveness of inhibition in COX-2/ VEGF pathway in tumor-associated angiogenesis together with lung metastasis.

\section{Results}

\section{Roles of COX-2 in Angiogenesis in Sponge Implant Model}

To ascertain the roles of inducible COX-2 and its downstream molecules responsible for the generation of prostaglandins in tumor-associated angiogenesis, we adopted a sponge implant model in the present study. This model is characterized by chronic hyperplastic inflammation and is designed to reflect tumorassociated angiogenesis by mimicking the angiogenic responses in the granulation tissues formed around the implants. When the circular sponge discs were implanted into the subcutaneous tissues of normal male ddy mice, the angiogenesis determined in terms of the hemoglobin content of the sponge granulation tissues occurred gradually (Fig. 1B). Direct topical injections of basic fibroblast growth factor (bFGF) enhanced the angiogenic reaction, judging from the degree of redness of the sponge implant (Fig. 1A). This treatment significantly facilitated angiogenesis in terms of hemoglobin concentration at Day 14 (Fig. 1B). Daily oral administration of the COX-2 inhibitor NS398 significantly suppressed the angiogenesis stimulated with bFGF. It was thus suggested that the angiogenesis observed in the sponge granulation tissues was enhanced in a COX-2-dependent fashion.

\section{Roles of VEGF in Angiogenesis and Induction Factors of VEGF in Sponge Implantation Model}

To examine the COX-2-dependent downstream molecules, we first tested the effects of topical injections of the neutralizing antibodies against several growth factors and against a cytokine into the sponge implants. The antibodies against epithelial growth factor (EGF), transforming growth factor (TGF)- $\beta$, and IL-1 did not reduce the angiogenesis at all, but by contrast, the VEGF antibody markedly suppressed the angiogenic reaction (Fig. 2A).

The expression of VEGF mRNA was not detectable in normal subcutaneous tissues in mice without sponge implantation (data not shown). The expression was certainly up-regulated in the sponge granulation tissue stimulated with bFGF (Fig. 2B). The upregulation of VEGF expression was suppressed with daily oral administration of NS398 (Fig. 2B).

Immunoreactive VEGF protein was also detectable by a specific ELISA in the exudates in the sponge implants stimulated with bFGF (Fig. 2C). This VEGF level was strongly reduced with topical injections of either an adenylate cyclase inhibitor, SQ22,536, and the protein kinase A inhibitor $\mathrm{H}-89$ (Fig. 2C). These results suggested that VEGF had a crucial role in the facilitation of angiogenesis in this model and that its induction was dependent on the COX-2 and adenylate cyclase-protein kinase A signaling pathway.

\section{Roles of COX-2 and VEGF in Tumor-Associated Angiogenesis}

Seeking to identify the roles of COX-2 in tumorassociated angiogenesis, we first tested the Millipore chamber model, in which angiogenesis was determined by the hemoglobin content of the granulation tissues formed around the tumor-cell-containing Millipore chambers. Implantation of the chambers containing sarcoma 180 (S-180) cells enhanced angiogenesis, compared with that seen in mice in which the chambers were implanted without tumor cells. Daily oral administration of aspirin or NS-398 strongly reduced angiogenesis (Fig. 3, A and B). The same was true in the granulation tissues formed around the chambers containing cells of another tumor cell line, Lewis lung carcinoma (LLC) (Fig. 3, C and D).

We next implanted the tumor cells into the subcutaneous tissues of mice and tested the angiogenesis in and around the tumors that developed (Fig. 4). The effects of COX inhibitors on tumor angiogenesis were first tested using S-180 cells. In control mice treated with vehicle solution, solid tumors were apparent 14 days after tumor cell implantation. Consistent with the marked red color of the tumors, angiogenesis was substantial in vehicle-treated mice (Fig. 4A). The extent of tumorinduced angiogenesis was assessed on the basis of hemoglobin content, which correlated well with the vascular density in the tumor under histologic examination (Fig. 4B). Daily oral administration of mofezolac, an inhibitor acting selectively on COX-1, had no significant effect on tumor-associated angiogenesis (Fig. 4C). In 
A

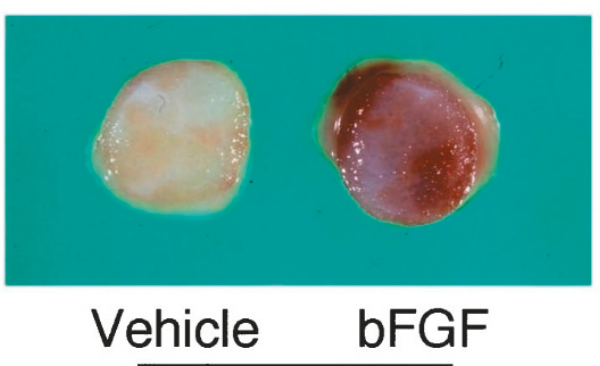

\section{Day 14}

B

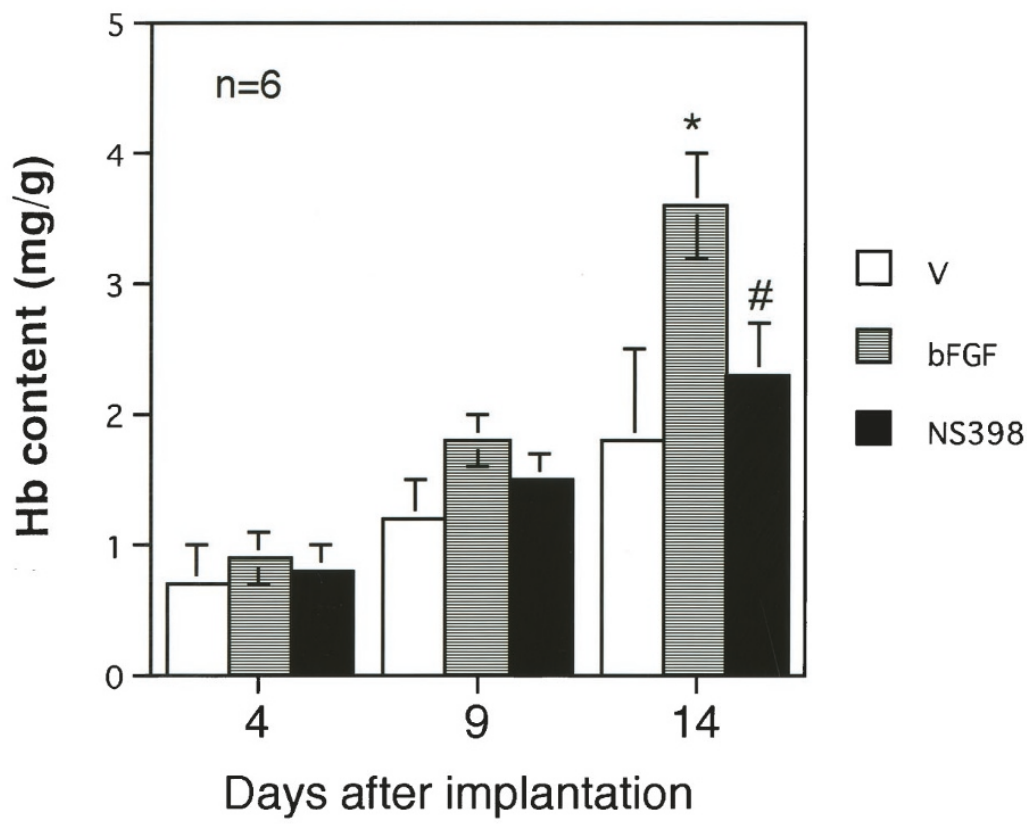

Figure 1.

Effect of a cyclo-oxygenase (COX)-2 inhibitor, NS-398, on angiogenesis in sponge-induced granulation tissues. (A) Typical appearance of sponge implants at Day 14 after excision from the back of ddy mice. Basic fibroblast growth factor (bFGF, $100 \mathrm{ng}$ ) was topically injected into the sponge matrix each day. (B) Time course of angiogenesis. Hemoglobin content of sponge granulation tissues was determined as a marker of angiogenesis at Days 4 , 9 , and 14 . $V$ : Physiological saline 100 $\mu \mathrm{l} /$ day/site was injected into the sponge implants, and vehicle solution ( $5 \%$ gum Arabic in distilled water) was given orally bid throughout the experiment. $b F G F$ : 100 ng of bFGF in $100 \mu$ l of physiological saline was injected into the implants, and vehicle solution (5\% gum Arabic in distilled water) was given orally throughout the experiment. NS398: $100 \mathrm{ng}$ of bFGF in $100 \mu \mathrm{l}$ of physiological saline was injected into the sponge implants, and NS-398 suspension in $5 \%$ gum Arabic was given $\left(10 \mathrm{mg} / \mathrm{kg}\right.$ ) orally bid throughout the experiment. Data are means \pm SEM for six sponges. ${ }^{*} p<0.05$ versus $\mathrm{V}$ (ANOVA). \#p $<0.05$ versus bFGF (ANOVA).

contrast, the COX-2-selective inhibitors JTE-522 and NS-398 significantly reduced tumor-related angiogenesis, as did aspirin, a nonselective COX inhibitor (Fig. 4C). These results suggested that COX-2 had a facilitating role in tumor-associated angiogenesis in this model, as well as in the Millipore chamber models. Further, we topically injected the neutralizing antibody against VEGF in this model (Fig. 4D). VEGF antibody significantly reduced tumor-associated angiogenesis in comparison with the angiogenesis in animals treated with control IgG (Fig. 4D).

\section{Roles of COX-2 and VEGF in Tumor Growth}

To test the effect of inhibition of COX on tumor growth, we implanted S-180 cell suspension in the subcutaneous tissue of mice and administered COX inhibitors daily
(Fig. 5A). In the vehicle control mice, substantial solid tumors developed during the 14-day experimental period. Daily oral administration of mofezolac, the inhibitor acting selectively on COX-1, had no significant effect on tumor growth. By contrast, the COX-2-selective inhibitors JTE-522 and NS-398 markedly suppressed tumor growth. Aspirin, a nonselective COX inhibitor, reduced tumor size to the same extent as these COX-2 inhibitors. VEGF antibody treatment significantly reduced tumor development, in comparison with control IgG treatment (Fig. 5B). These results suggested that COX-2NEGF had a facilitating role in tumor development.

\section{Discussion}

Angiogenesis is believed to be an important factor in the promotion of tumor growth (Folkman, 1971, 1996). 


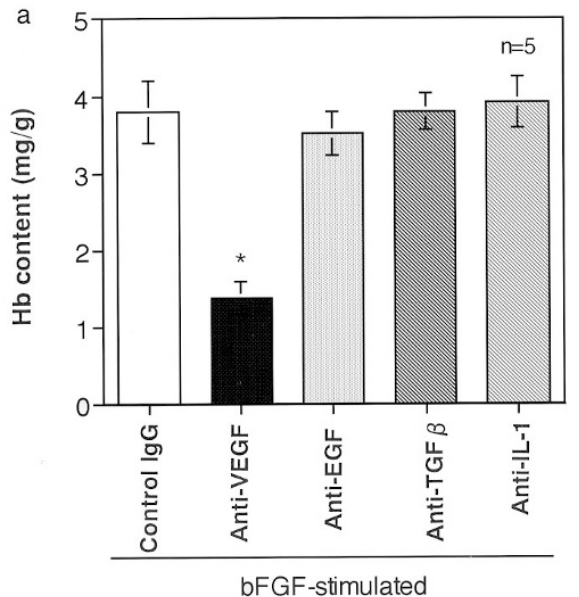

b

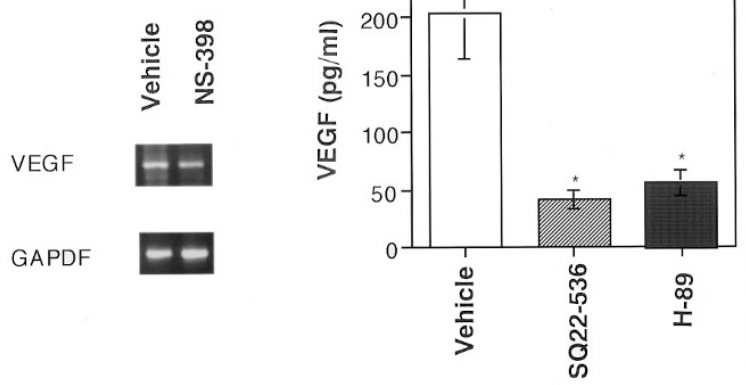

Figure 2.

Effects of topical injections of neutralizing antibodies against the growth factors on sponge angiogenesis and identification of the factor modulating vascular endothelial growth factor (VEGF) expression. (A) In separate tests, antibodies to VEGF, epithelial growth factor (EGF), transforming growth factor (TGF) $\beta$, and IL-1 were topically injected daily into the sponge matrix (2 $\mu \mathrm{g} / \mathrm{site} /$ day) for 14 days. The sponge angiogenesis was topically stimulated with basic fibroblast growth factor (bFGF) (100 ng/site/day). Data are means \pm SEM for five sponges. Control IgG: Mice receiving nonimmune IgG fraction. * $p$ $<0.05$ versus control IgG (ANOVA). (B) RT-PCR analysis of VEGF in sponge granulation tissues dissected at Day 14 from mice receiving either vehicle solution (5\% gum Arabic) or NS-398 suspension $(10 \mathrm{mg} / \mathrm{kg}$, administered orally bid). The sponge angiogenesis was topically stimulated with bFGF (100 ng/site/day). (C) VEGF levels determined with specific ELISA. The sponge implants were topically stimulated with bFGF (100 ng/site/day) for 14 days. SQ22,536 and $\mathrm{H}-89$, which are inhibitors of adenylate cyclase and protein kinase $\mathrm{A}$, respectively, were dissolved in physiological saline containing $1 \%$ (V/V) dimethyl sulfoxide (DMSO) and were topically administered (10 $\mu \mathrm{g} / \mathrm{site}$ / day, once a day) throughout the experimental period. For the control mice, physiological saline containing DMSO was injected topically. Data are means \pm SEM for six sponges. ${ }^{\star} p<0.05$ versus vehicle control (ANOVA).

Substantial increases in tumor mass must be preceded by an increase in blood supply to provide the nutrients and oxygen required for tumor growth. It is obvious that the vessels newly formed to supply blood to the tumors are derived from the pre-existing vascular bed in the host. The mechanisms for promotion of tumor-associated angiogenesis are suggested to be activated in the early stages of tumor development (Hanahan and Folkman, 1996). In the present experiment, we have shown with our S-180 implantation model and Millipore chamber experiment that COX-2selective inhibitors strongly inhibit tumor-associated angiogenesis, suggesting the significant roles of COX-2 in angiogenic responses seen in both models.
The COX-2 blockers and the nonselective COX inhibitor aspirin inhibited tumor growth by $\sim 80 \%$, which was similar in extent of the effect of such inhibitors on the growth of COX-2-overexpressing human tumor cells transplanted into nude mice described in a previous report (Tsujii et al, 1998). Because COX-2 has been reported to be localized in both tumor cells and the adjacent stromal cells, PGs generated through the presence of COX-2 might act on the tumor cells (a cell autonomous effect) or on the surrounding stroma cells (a cell nonautonomous or landscaping effect) to facilitate tumor development (Gupta and Dubois, 2001; Kinzler and Vogelstein, 1998). As an example of the former effect, rat intestinal epithelial cells, which were engineered to overexpress COX-2, showed high levels of the antiapoptotic protein BCL-2 (Tsujii and Dubois, 1995) and the COX-2-overexpressing Caco-2 cells showed increased cell migration and invasion, activities that are closely associated with several of the matrix metalloproteinases (Tsujii et al, 1997). As an example of the latter effect, we have focused on the ability of COX-2-derived PGs to stimulate the angiogenesis associated with tumors. Angiogenesis in vivo is maintained by endogenous growth factors. It was reported previously that the PGs derived from COX2-overexpressing Caco-2 cells acted on the tumor cells themselves and secreted several growth factors that synergistically enhanced tube formation by cocultured endothelial cells in vitro (Tsujii et al, 1998). If this should also occur in vivo, the tumor cells would facilitate angiogenesis in an autocrine fashion. However, the same group reported separately using COX-2 knockout mice that in addition to COX-2 expression in tumor cells, expression in the stroma cells surrounding the tumors also has a crucial role in the enhancement of the angiogenesis in a tumor implantation model (Williams et al, 2000). If the latter mechanism is important in vivo, tumor-associated angiogenesis would depend more on COX-2 in the stroma than on that in the tumor cells.

To test the proangiogenic capacity COX-2 in the stroma, we developed the sponge implantation model in which the granulation tissues developed gradually with very active angiogenesis, and this model may mimic the events during the development of the stroma (Hayashi et al, 2002; Majima et al, 1997, 2000; Muramatsu et al, 2000). This model possesses several advantages for studies of angiogenesis. Angiogenesis can be readily quantified by measurement of the hemoglobin content of the sponge-induced granulation tissue together with histologic examination, and the endogenous substances that induce or inhibit angiogenesis are readily determined. Further, the effects of exogenous substances can be investigated on direct injection into the sponge (Hayashi et al, 2002; Majima et al, 1997, 2000; Muramatsu et al, 2000). Judging from the results shown in Figures 1 and 2, the angiogenesis observed in this model was dependent on COX-2 but not on COX-1, and the growth factor that induced or maintained angiogenesis is VEGF but not EGF, TGF $\beta$, or IL-1 (Fig. 2A). VEGF mRNA was detectable in these sponge implants, and its expression was up-regulated by COX-2-mediated PGs, 
A
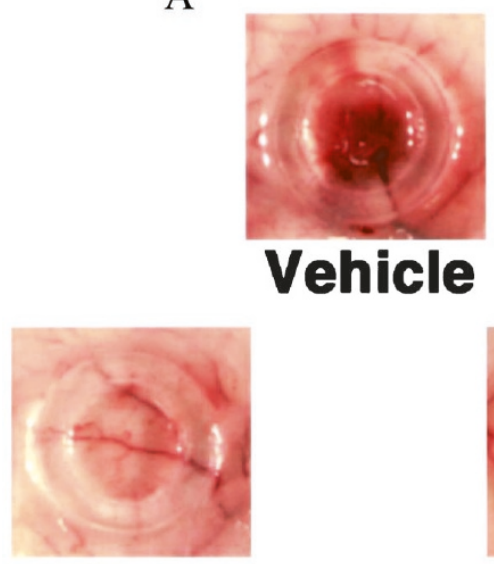

Aspirin

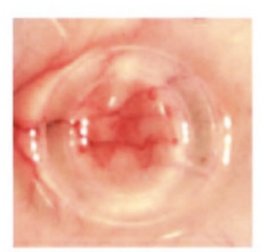

NS398
B

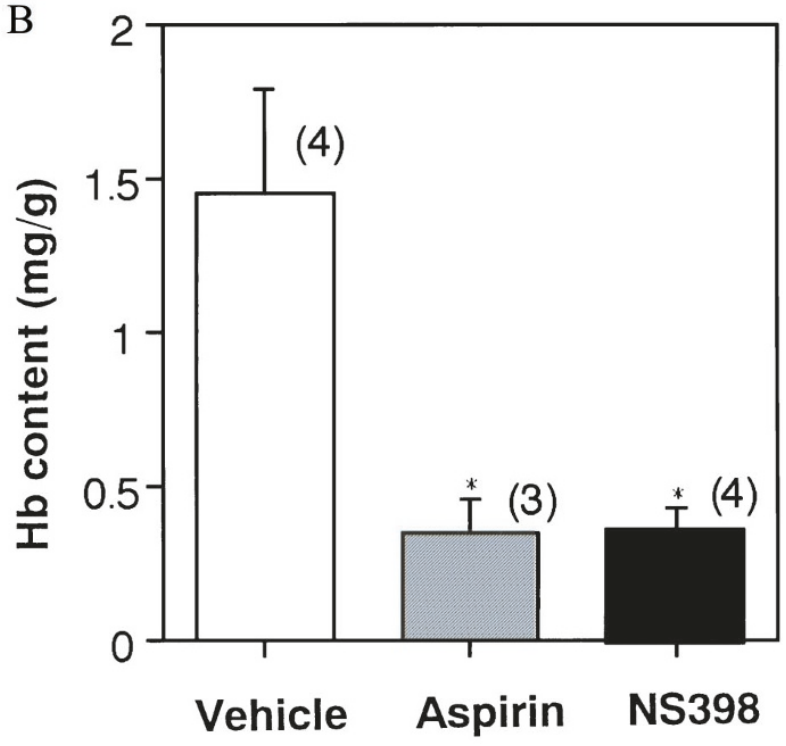

D

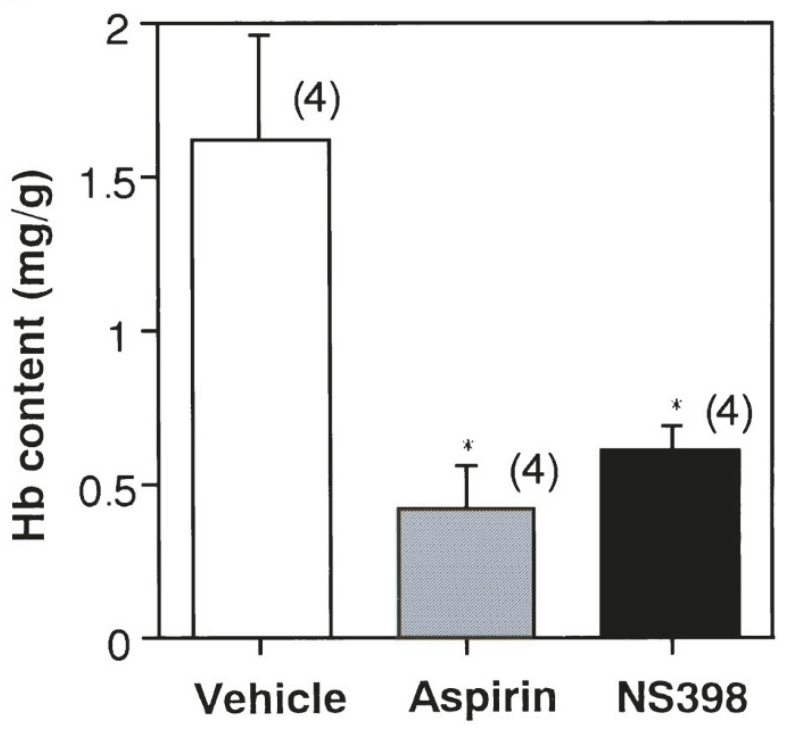

\section{Figure 3.}

Effects of cyclo-oxygenase (COX) inhibitors on angiogenesis formed around Millipore chambers containing tumor cells. (A) Typical appearance of granulation tissues formed around Millipore chambers containing sarcoma 180 cells. Aspirin or NS-398 suspension in 5\% gum Arabic in distilled water was administered (10 mg/kg) orally bid for 5 days. Vehicle mice were given only vehicle solution ( $5 \%$ gum Arabic in distilled water). (B) Hemoglobin content of granulation tissues formed around the Millipore chambers (sarcoma 180 cells). Data (Day 5) are means \pm SEm for the numbers of chambers indicated in parentheses. ${ }^{\star} p<0.05$ versus vehicle-treated mice (ANOVA). (C) Typical appearance of granulation tissues formed around Millipore chambers containing Lewis lung carcinoma (LLC) cells. (D) Hemoglobin content of granulation tissues formed around Millipore chambers (LLC cells). Data (Day 5 ) are means \pm sEm for the numbers of chambers indicated in parentheses. ${ }^{*} p<$ 0.05 versus vehicle-treated mice (ANOVA).

because NS-398, which selectively inhibited COX-2, certainly reduced the expression of VEGF mRNA (Fig. 2B). PGs generated by COX-2 were not identified in the present experiments, but immunoreactive VEGF levels in the sponge matrix were reduced by adenylate cyclase inhibitor and protein kinase A inhibitor (Fig. 2C), suggesting that $P G$ signaling was responsible for the elevation of CAMP. PGE2 receptors have four subtypes, of which EP2, EP4, and some splicing variant of EP3 receptor are known to activate adenylate cyclase and to elevate intracellular cAMP levels (Narumiya et al, 1999). IP receptor may also participate this enhancement of angiogenesis, because IP receptor is linked to the activation of adenylate cyclase (Narumiya et al, 1999). COX-2-derived $\mathrm{PGs}$, which are linked to the adenylate cyclase activation, may have a crucial role in the sponge model mimicking the stromal response.

If the same mechanism operates in tumor development in vivo, the inhibition of COX-2 or VEGF, or both, may be sufficiently potent to suppress the tumor- 
A

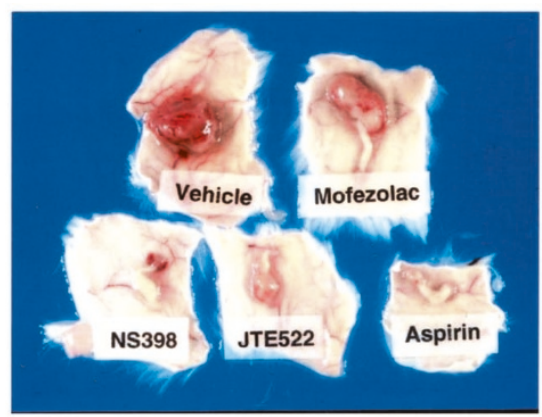

B

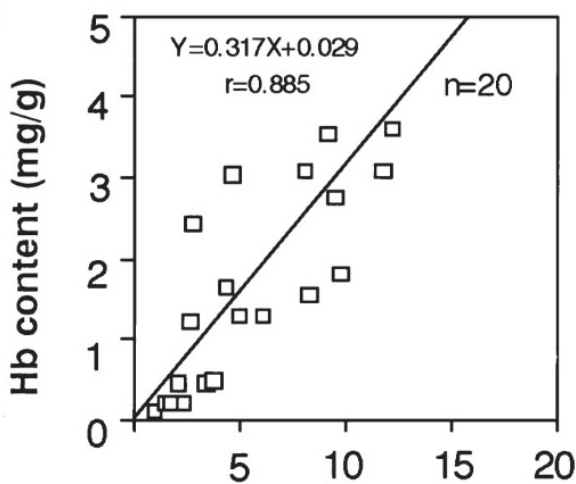

Relative density of microvessels (\%)
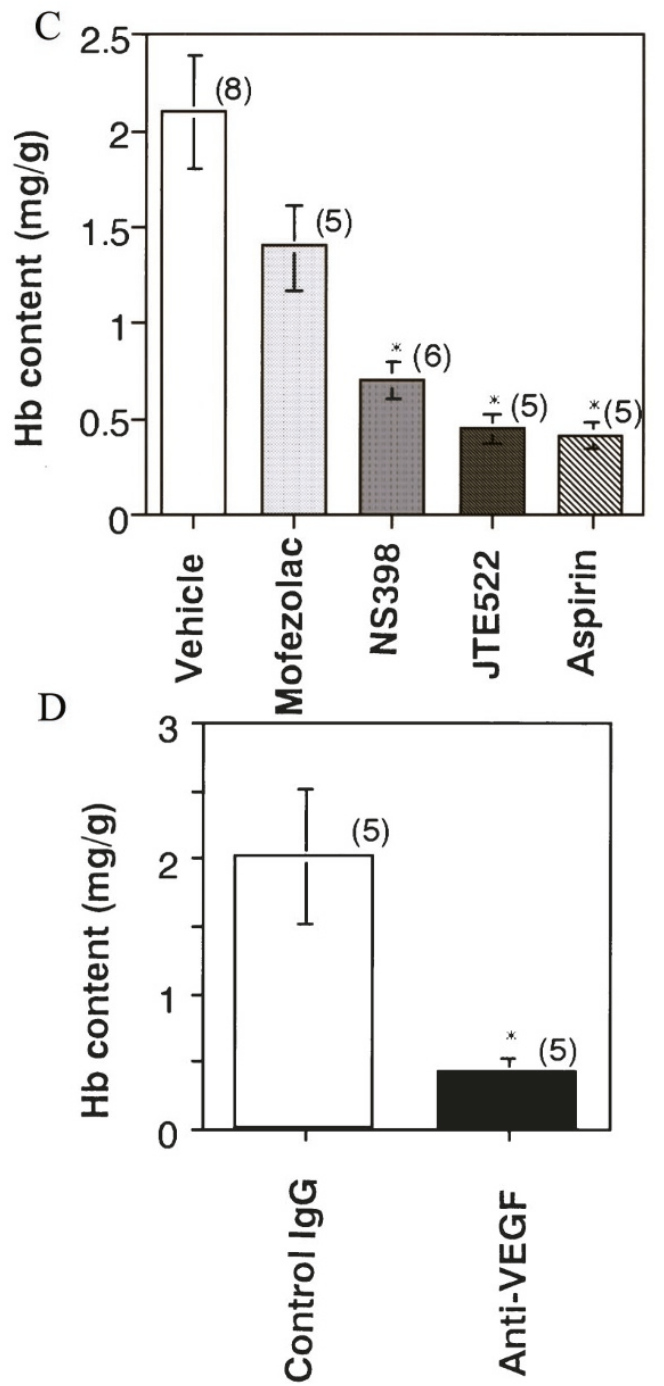

associated angiogenesis and tumor growth. In fact, the proangiogenic responses observed in the chamber model, which may mimic the angiogenesis in the tumor stroma, were certainly reduced with a COX-2 inhibitor (Fig. 3). This inhibition was consistently observed in two different cell lines. Further, the tumorassociated angiogenesis in the tumor implantation model was markedly reduced by COX-2 inhibitors and by aspirin (a nonselective COX inhibitor) but not by a COX-1 selective inhibitor (Fig. 4). Angiogenesis in this model also suppressed by a VEGF-neutralizing antibody (Fig. 4). Tumor growth was also COX-2/NEGFdependent (Fig. 5). These results taken together indicate that COX-2-mediated PG formation and the up-regulation of VEGF as a downstream molecule of COX-2 may both have roles in the in vivo tumor implantation model. COX-2 inhibitor effectively suppressed tumor-associated angiogenesis and consequently reduced tumor growth. The host microenvironment is thought to influence tumor progression (Fukumura et al, 1997; Gohongi et al, 1999). Immunohistochemical examination of human colorectal cancer tissue has revealed that the marked COX-2 expression was detectable not only in tumor cells but also in inflammatory cells and fibroblasts in the stroma (Harada et al, 1994). It was also reported that COX-2 is abundant in the stromal cells that surround intestinal polyps in mice with a mutated APC gene (Ohshima et al, 1996). In our preliminary experiments, we detected marked VEGF immunoreactivity in the host stromal cells, including the fibroblast-like cells that surrounded the implanted sarcoma, although the immunoreactive COX-2 was localized in the tumor cells and stroma cells in S-180 implantation models. Thus, it is highly plausible that PGs derived from COX-2 may act preferentially on the stromal cells.

In conclusion, the present results demonstrate the significance of the COX-2-dependent pathway in tumor-associated angiogenesis and tumor growth. Further, we identified the downstream molecules that may respond to COX-2 to be VEGF. VEGF actually contributed as a promotor of tumor growth and tumorassociated angiogenesis. COX-2 inhibitors together with some agents that will block the COX-2/VEGFdependent pathway will become useful therapeutic tools against malignant solid tumors.

\section{Figure 4.}

Effects of cyclo-oxygenase (COX) inhibitors on tumor-associated angiogenesis. (A) Typical appearance of tumors. A suspension of sarcoma 180 cells was injected into the subcutaneous tissue of ddy mice. COX inhibitors (mofezolac, NS-398, JTE-522, and aspirin, each at a dose of $10 \mathrm{mg} / \mathrm{kg}$ ) were separately administered orally as suspensions $(0.05 \mathrm{ml}$ per $10 \mathrm{~g}$ of body mass) every 12 hours beginning on the day of cell implantation and continuing throughout the 14-day experimental period. Tumors were then dissected and photographed. (B) Correlation between the microvessel density and the hemoglobin content of tumor tissue. (C) Effects of COX inhibitors on tumor-associated angiogenesis at the end of the 14-day experimental period. Data are means \pm SEM for the numbers of tumors indicated in parentheses. ${ }^{*} p<0.05$ versus vehicletreated mice (ANOVA). (D) Effects of modulating vascular endothelial growth factor (VEGF) neutralizing antibody on tumor-associated angiogenesis at the end of the 14-day experimental period. Data are means \pm SEM for five mice. Control IgG: Mice receiving nonimmune IgG fraction. * $p<0.05$ versus control $\lg G$ (ANOVA). 

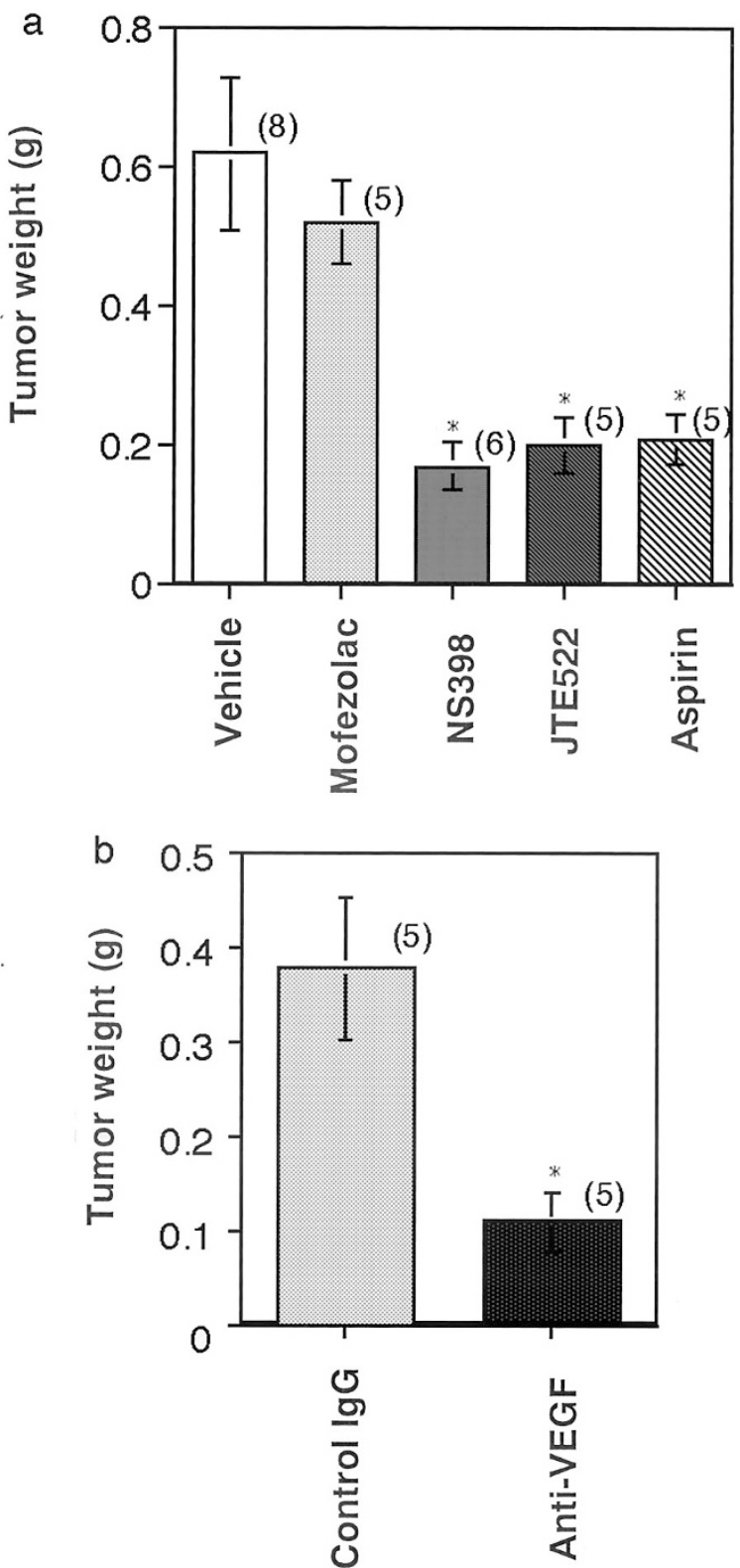

Figure 5.

(A) Effects of cyclo-oxygenase (COX) inhibitors on tumor growth at the end of the 14-day experimental period. A suspension of sarcoma 180 cells was injected into the subcutaneous tissue of ddy mice. COX inhibitors (mofezolac, NS-398, JTE-522, and aspirin, each at a dose of $10 \mathrm{mg} / \mathrm{kg}$ ) were separately administered orally bid (every 12 hours) beginning on the day of cell implantation and continuing throughout the 14-day experimental period. Tumors were then dissected and weighed. Data are means \pm SEM for the number of tumors indicated in parentheses. ${ }^{*} p<0.05$ versus vehicle-treated mice (ANOVA). (B) Effects of modulating vascular endothelial growth factor (VEGF) neutralizing antibody on tumor growth at the end of the 14-day experimental period. Data are means \pm SEM for five mice. Control IgG: Mice receiving nonimmune IgG fraction. ${ }^{\star} p<0.05$ versus control IgG (ANOVA).

\section{Materials and Methods}

\section{Animals}

Male ddy mice (7 weeks old) were obtained from SLC (Hamamatsu, Japan), and male C57/BL6 mice (7 weeks old) were obtained from CLEA Japan, Inc. (Tokyo, Japan). All mice were housed at a controlled humidity of $60 \pm 5 \%$ and a temperature $25 \pm 1^{\circ} \mathrm{C}$, with a 12-hour light/dark cycle. All animal experiments were performed in accordance with the guidelines for animal experiments of Kitasato University School of Medicine.

\section{Sponge Implantation Model of Angiogenesis}

Sponge disks (thickness, $5 \mathrm{~mm}$; diameter, $1.3 \mathrm{~cm}$ ) (Hayashi et al, 2002; Majima et al, 1997, 2000; Muramatsu et al, 2000) were implanted under light ether anesthesia into the subcutaneous tissues of the back of ddy mice. After a fixed number of days, the granulation tissues that had formed around the sponge were taken immediately after death induced by the ip administration of excessive doses of sodium pentobarbital. Neovascularization was assessed by the method described below.

bFGF (100 ng, Genzyme Corporation, Cambridge, Massachusetts) was dissolved in physiological saline $(0.1 \mathrm{ml})$ and topically injected into the sponge implants once a day to facilitate angiogenesis.

Neutralizing antibody against mouse VEGF (IgG, Genzyme) (Ishihara et al, 2002), which can block the biological activity of recombinant mouse VEGF164 and VEGF120 with the neutralizing dose $_{50}$, was used. One-hundred $\mathrm{ng} / \mathrm{ml}$, determined in the presence of 10 $\mathrm{ng} / \mathrm{ml}$ of mouse VEGF164, was dissolved in physiological saline $(5 \mu \mathrm{g} / 0.1 \mathrm{ml})$ and was topically administered to the sponge implants once a day $(5 \mu \mathrm{g} / \mathrm{site} /$ day). Other antibodies against IL-1, TGF $\beta$, and EGF were purchased from R\&D Systems Inc. (Minneapolis, Minnesota) and were topically injected into the sponge implants $(5 \mu \mathrm{g} / \mathrm{site} /$ day). For control mice, a nonimmune IgG fraction (Genzyme, $5 \mu \mathrm{g} / \mathrm{site} /$ day) was administered topically.

SQ22,536 (Research Biochemicals International, Natick, Massachusetts) and H-89 (Sigma, St. Louis, Missouri), which are inhibitors for adenylate cyclase and protein kinase A, respectively, were dissolved in physiological saline containing 1\% (VN) DMSO at a concentration of $10 \mu \mathrm{g} / 0.1 \mathrm{ml}$ and were topically administered (10 $\mu \mathrm{g} / \mathrm{site} /$ day) once a day throughout the experimental period. For the control mice, physiological saline containing 1\% DMSO was topically injected.

A COX-2 selective inhibitor, NS-398, was suspended with $5 \%$ gum Arabic at a concentration of 2 $\mathrm{mg} / \mathrm{ml}$ and was orally administered $(0.05 \mathrm{ml} / 10 \mathrm{~g}$ body weight, $10 \mathrm{mg} / \mathrm{kg}$, bid) to the mice. For vehicle control mice, distilled water with $5 \%$ gum Arabic was administered orally $(0.05 \mathrm{ml} / 10 \mathrm{~g}$ body weight, bid).

\section{Tumor Cells}

Murine S-180 and LLC cells were provided by Dainippon Pharmaceutical Company (Osaka, Japan). These cells were cultured in Dulbecco's modified Eagle's medium supplemented with $10 \%$ fetal bovine serum, penicillin (100 units/ml) (Fujita et al, 2002), and streptomycin (100 units $/ \mathrm{ml}$ ), all of which were obtained from Gibco BRL, Life Technologies, Inc. (Rockville, Maryland). 


\section{Millipore Chamber Experiment}

S-180 cells and LLC cells were washed with PBS solution (PBS; $\mathrm{NaCl}$ at $8 \mathrm{~g} / \mathrm{l}, \mathrm{KCl}$ at $0.2 \mathrm{~g} / \mathrm{l}$, $\mathrm{Na}_{2} \mathrm{HPO}_{4} 12 \mathrm{H}_{2} \mathrm{O}$ at $2.9 \mathrm{~g} / \mathrm{l}, \mathrm{KH}_{2} \mathrm{PO}_{4}$ at $0.2 \mathrm{~g} / \mathrm{l}, \mathrm{pH}$ 7.2), and were suspended in PBS at a concentration of $1 \times 10^{8} \mathrm{cells} / \mathrm{ml}$. A Millipore chamber (filter pore size $0.45 \mu \mathrm{m}$, Millipore Company, Bedford, Massachusetts) was filled with $0.2 \mathrm{ml}$ of one of the cell suspensions and was implanted subcutaneously into the dorsum of ddy mice (Hayashi et al, 2002; Iwahana et al, 1996). Five days after the implantation, the granulation tissues that had formed on the Millipore chamber were taken immediately after death had been induced by excess doses of sodium pentobarbital. After the granulation tissues were excised, the hemoglobin concentrations in the granulation tissues were determined.

NS-398 or aspirin was administered orally $(10 \mathrm{mg} /$ $\mathrm{kg}$, bid) to the mice bearing the chambers, as mentioned above. For vehicle control mice, distilled water with $5 \%$ gum Arabic was given orally.

\section{Tumor Implantation Models}

S-180 cells were harvested and washed three times with PBS. The cells were pelleted by brief centrifugation at $300 \times g$ at $25^{\circ} \mathrm{C}$. The supernatant was aspirated, and the cells were resuspended in PBS at a density of $1 \times 10^{7}$ cells $/ \mathrm{ml}$, and $100 \mu$ l of the resulting suspension was injected into the subcutaneous tissue of the flank of ddy mice.

Fourteen days after the injection, ddy mice injected with S-180 cells were killed with an excess dose of sodium pentobarbital, and tumor tissues were excised carefully. The total weights of the excised tumor tissues were measured immediately after harvesting. Angiogenesis was assessed by the method described below.

A selective COX-1 inhibitor (mofezolac), a selective COX-2 inhibitor (JTE522), NS-398, or aspirin was administered orally $(10 \mathrm{mg} / \mathrm{kg}$, bid) to mice injected with S-180 cells, as mentioned above. To the vehicle control mice, distilled water with $5 \%$ gum Arabic was given orally.

In a separate experiment, neutralizing antibody against mouse VEGF (Genzyme) was administered topically in the vicinity of the tumors formed on the back of mice (once a day, $2 \mu \mathrm{g} / \mathrm{site} /$ day). For control rats, a nonimmune IgG fraction (Genzyme) was administered topically.

\section{Determination of Angiogenesis}

Neovascularization was assessed by measuring the concentration of hemoglobin in the granulation tissues formed around the sponge implants (Majima et al, 2000), Millipore chambers (Hayashi et al, 2002), or in the tumor tissues, including the surrounding stroma (Gatto et al, 1999; Kerr et al, 1999; Zeng et al, 2000). Briefly, after the granulation tissues from the sponge or the Millipore chamber were weighed, they were cut into several pieces with scissors. Distilled water 4 times the weight of the sample granulation tissues was added to each sample, which was then homogenized with a Polytrone homogenizer (Kinematica $\mathrm{GmbH}$, Lucerne, Switzerland). The hemoglobin concentration in the supernatant after centrifugation at $5000 \times g$ was determined using a hemoglobin assay kit (Hemoglobin B Testwako, Wako Pure Chemical Industries Ltd., Osaka, Japan). The concentrations of hemoglobin in the granulation tissues were expressed as $\mathrm{mg} / \mathrm{g}$ wet tissue.

Microvessel density in the tumor and stroma was assessed as a parameter of tumor-associated angiogenesis according to the established methods described previously (Borre et al, 1998; Fujita et al, 2002; Weidner et al, 1991). The individual microvessels were counted in a $\times 200$ field microscope. CD-31 immunoreactive endothelial cells were clearly and easily differentiated from the tumor cells and other connective tissue elements. Relative microvessel density was expressed in terms of relative microvessel area, which was calculated with the image analysis software National Institutes of Health Image (NIH Research Service Branch) and was expressed as the percentage area of vessels per observed area $\left(\mathrm{mm}^{2} / \mathrm{mm}^{2} \times 100\right)$ (Fujita et al, 2002).

\section{RT-PCR}

Transcripts encoding VEGF and glyceraldehyde-3phosphate dehydrogenase (GAPDH) were quantified by RT-PCR analysis. Sponge granulation tissue was removed and rapidly frozen in liquid nitrogen. The frozen tissue was pulverized in a stainless steel cylinder cooled with liquid nitrogen. Total RNA was extracted from the tissue with Isogen (Wako), and cDNA was synthesized from $1 \mu \mathrm{g}$ of total RNA with the use of an oligo-p(dT)15 primer and AMV reverse transcriptase (Boehringer Mannheim, Mannheim, Germany). Fifty ng of cDNA was amplified with $1 \mathrm{U}$ of TaqDNA polymerase in a 25- $\mu$ l reaction mixture containing 10 $\mathrm{mm}$ Tris- $\mathrm{HCl}$ (pH 8.3), $50 \mathrm{~mm} \mathrm{KCl,} 1.5 \mathrm{~mm} \mathrm{MgCl}$, and $0.2 \mu \mathrm{M}$ deoxynucleoside triphosphate, and each of the forward and reverse primers at $0.6 \mu \mathrm{M}$. The amplification protocol comprised 25 cycles (VEGF) or 20 cycles (GAPDH) of 45 seconds at $94^{\circ} \mathrm{C}, 60$ seconds at $55^{\circ} \mathrm{C}$, and 60 seconds at $72^{\circ} \mathrm{C}$. The reaction mixtures were subsequently applied to a $2 \%$ agarose gel, and the amplified products were stained with ethidium bromide. The primers used were as follows: 5'-AACCATGAACTTTCTGCTCTC-3' (sense) and 5'-GTGATTTTCTGGCTTTGTTC-3' (antisense) for VEGF, and 5'-CCCTTCATTGACCTCAACTACAATGGT-3' (sense), and 5'-GAGGGGCCATCCACAGTCTTCTG-3' (antisense) for GAPDH.

\section{Determination of VEGF Levels in Fluids in Sponge Matrix}

Under light ether anesthesia, the fluids in the sponge matrix were collected by aspiration with a $26-\mathrm{G}$ needle and were centrifuged at $2000 \times g$ for 20 minutes. The resulting supernatant was diluted with the assay buffer in ELISA for VEGF, and the levels were determined 
with the specific ELISA for mouse VEGF (Genzyme) (Amano et al, 2001). The levels of VEGF were expressed in terms of picograms/milliliters in the collected fluids.

\section{Chemicals and Other Materials}

The aspirin was a gift from Merck (Rahway, New Jersey); and NS-398 (N-[2-(cyclohexyloxy)-4-nitrophenyl]methanesulfonamide) (Futaki et al, 1993) was purchased from Cayman Chemical (Ann Arbor, Michigan). The mofezolac ([3,4-di(4-methoxyphenyl)-5-isoxazolyl] acetic acid) (Kitamura et al, 2002) was a gift from Yoshitomi Pharmaceutical Corporation. (Tokyo, Japan). JTE-522 (4-[4-cyclohexyl-2-methyloxazol-5-yl]-2-fluorobenzenesulfonamide) (Wakitani et al, 1998) was donated by Japan Tobacco Inc. (Tokyo, Japan). The hemoglobin assay kit was obtained from Wako Pure Chemical Industries Ltd. (Osaka, Japan). All other substances used were of analytical grade and were purchased from commercial sources.

\section{Statistical Analysis}

Data are expressed as means \pm SEM. Comparisons among multiple groups were performed by factorial ANOVA (ANOVA) followed by Scheffe's test. Comparisons between two groups were performed with Student's $t$ test. A $p$ value of $<0.05$ was considered statistically significant.

\section{Acknowledgements}

We thank Michiko Ogino, Keiko Nakamigawa, Osamu Katsumata, and Masaki Soma for their technical assistance and C.W.P. Reynolds for correcting the English of this manuscript.

\section{References}

Amano H, Ando K, Minamida S, Hayashi I, Ogino M, Yamashina S, Yoshimura $H$, and Majima M (2001). Adenylate cyclase/protein kinase: A signaling pathway enhances angiogenesis through induction of vascular endothelial growth factor in vivo. Jpn J Pharmacol 87:181-188.

Borre M, Offersen BV, Nerstrøm B, and Overgaard J (1998). Microvessel density predicts survival in prostate cancer patients subjected to watchful waiting. $\mathrm{Br} \mathrm{J}$ Cancer 78:940944.

Folkman J (1971). Tumor angiogenesis: Therapeutic implications. N Engl J Med 285:1182-1186.

Folkman $J$ (1996). What is the evidence that tumors are angiogenesis dependent? J Natl Cancer Inst 82:4-6.

Fujita M, Hayashi I, Yamashina S, Itoman M, and Majima M (2002). Blockade of angiotensin AT1a receptor signaling reduces tumor growth, angiogenesis, and metastasis. Biochem Biophys Res Commun 294:441-447.

Fukumura D, Yuan F, Monsky WL, Chen Y, and Jain RK (1997). Effect of host microenvironment on the microcirculation of human colon adenocarcinoma. Am J Pathol 151:679688.
Futaki N, Yoshikawa K, Hamasaka Y, Arai I, Higuchi S, lizuka $\mathrm{H}$, and Otomo S (1993). NS-398, a novel non-steroidal anti-inflammatory drug with potent analgesic and antipyretic effects, which causes minimal stomach lesions. Gen Pharmacol 24:105-110.

Gatto C, Rieppi M, Borsotti P, Innocenti S, Ceruti R, Drudis T, Scanziani E, Casazza AM, Taraboletti G, and Giavazzi R (1999). BAY 12-9566, a novel inhibitor of matrix metalloproteinases with antiangiogenic activity. Clin Cancer Res 5:3603-3607.

Gohongi T, Fukumura D, Boucher Y, Yun CO, Soff GA, Compton C, Todoroki T, and Jain RK (1999). Tumor-host interactions in the gallbladder suppress distal angiogenesis and tumor growth: Involvement of transforming growth factor beta. Nature Med 5:1203-1208.

Gupta RA and Dubois RN (2001). Colorectal cancer prevention and treatment by inhibition of cyclooxygenase-2. Nat Rev Cancer 1:11-21.

Hanahan D and Folkman J (1996). Patterns and emerging mechanisms of angiogenic switch during tumorigenesis. Cell 86:353-364.

Harada S, Nagy JA, Sullivan KA, Thomas KA, Endo N, Rodan GA, and Rodan SB (1994). Induction of vascular endothelial growth factor expression by prostaglandin $E_{2}$ and $E_{1}$ in osteoblasts. J Clin Invest 93:2490-2496.

Hayashi I, Amano H, Yoshida S, Kamata K, Kamata M, Inukai M, Fujita T, Kumagai $Y$, Furudate $S$, and Majima M (2002). Suppressed angiogenesis in kininogen-deficiencies. Lab Invest $82: 871-80$

Ishihara K, Kamata M, Hayashi I, Yamashina S, and Majima $M$ (2002). Roles of bradykinin in vascular permeability and angiogenesis in solid tumor. Int Immunopharmacol 2:499509 .

Iwahana M, Nakayama Y, Tanaka NG, Goryo M, and Okada K (1996). Quantification of tumour-induced angiogenesis by image analysis. Int J Exp Pathol 77:109-114.

Kerr JS, Wexler RS, Mousa SA, Robinson CS, Wexler EJ, Mohamed S, Voss ME, Devenny JJ, Czerniak PM, Gudzelak A Jr, and Slee AM (1999). Novel small molecule alpha v integrin antagonists: Comparative anti-cancer efficacy with known angiogenesis inhibitors. Anticancer Res 19:959-968.

Kinzler KW and Vogelstein B (1998). Landscaping the cancer terrain. Science 280:1036-1037.

Kitamura T, Kawamori T, Uchiya N, Itoh M, Noda T, Matsuura M, Sugimura T, and Wakabayashi K (2002). Inhibitory effects of mofezolac, a cyclooxygenase-1 selective inhibitor, on intestinal carcinogenesis. Carcinogenesis 23:1463-1466.

Majima M, Hayashi I, Muramatsu M, Katada J, Yamashina S, and Katori M (2000). Cyclooxygenase-2 enhances basic fibroblast growth factor-induced angiogenesis through the induction of vascular endothelial growth factor in rat sponge implants. Br J Pharmacol 130:641-649.

Majima M, Isono M, Ikeda Y, Hayashi I, Hatanaka K, Harada Y, Katsumata O, Yamashina S, Katori M, and Yamamoto S (1997). Significant roles of inducible cyclooxygenase (COX)-2 in angiogenesis in rat sponge implants. Jpn J Pharmacol 75:105-114.

Marx J (2001). Cancer research. Anti-inflammatories inhibit cancer growth: But how? Science 291:581-582. 
Muramatsu M, Katada J, Hayashi I, and Majima M (2000). Chymase as a proangiogenic factor. A possible involvement of chymase-angiotensin-dependent pathway in the hamster sponge angiogenesis model. J Biol Chem 275:5545-5552.

Narumiya S, Sugimoto Y, and Ushikubi F (1999). Prostanoid receptors: Structures, properties, and function. Physiol Rev 79:1193-1226.

Ohshima M, Dinchuk JE, Kargman SL, Oshima H, Hancock B, Kwong E, Trzaskos JM, Evans JF, and Taketo MM (1996). Suppression of intestinal polyposis in $A p c \Delta^{716}$ knockout mice by inhibition of cyclooxygenase 2 (COX-2). Cell 87:803809.

Prescott SM and White RL (1996). Self-promotion? Intimate connections between APC and prostaglandin $\mathrm{H}$ synthase-2. Cell 87:783-786.

Shiff SJ and Rigas B (1997). Nonsteroidal anti-inflammatory drugs and colorectal cancer: Evolving concepts of their chemopreventive actions. Gastroenterology 113:1992-1998.

Smalley W and Dubois RN (1997). Colorectal cancer and non-steroidal anti-inflammatory drugs. Adv Pharmacol 39:120.

Tsujii M and Dubois RN (1995). Alterations in cellular adhesion and apoptosis in epithelial cells overexpressing prostaglandin endoperoxide synthase-2. Cell 83:493-501.
Tsujii M, Kawano S, and Dubois RN (1997). Cyclooxygenase-2 expression in human colon cancer cells increases metastatic potential. Proc Natl Acad Sci USA 94:3336-3340.

Tsujii M, Kawano S, Tsuji S, Sawaoka H, Hori H, and Dubois RN (1998). Cyclooxygenase regulates angiogenesis induced by colon cancer cells. Cell 93:705-716.

Wakitani K, Nanayama T, Masaki M, and Matsushita M (1998). Profile of JTE-522 as a human cyclooxygenase-2 inhibitor. Jpn J Pharmacol 78:365-371.

Weidner N, Semple JP, Welch WR, and Folkman J (1991). Tumor angiogenesis and metastasis-correlation in invasive breast carcinoma. New Engl J Med 324:1-8.

Williams CS, Tsujii M, Reese J, Dey SK, and Dubois RN (2000). Host cyclooxygenase-2 modulates carcinoma growth. J Clin Invest 105:1589-1594.

Zeng G, Gao L, Birkle S, and Yu RK (2000). Suppression of ganglioside GD3 expression in a rat F-11 tumor cell line reduces tumor growth, angiogenesis, and vascular endothelial growth factor production. Cancer Res 60:6670-6676. 